\title{
DEMOGRAPHIC FACTORS ASSOCIATED WITH MALARIA PREVALENCE IN ZARIA, KADUNA STATE, NIGERIA
}

Benjamin, G.Y., Kanai, E. T., Moses, B. E. and Aluwong, G.S.

Department of Agricultural Technology, School of Agricultural Technology, Nuhu Bamalli

Polytechnic, Zaria.

Corresponding author: gideonbenjamin.y@gmail.com

\section{ABSTRACT}

Malaria is a parasitic disease, endemic to Nigeria and many tropical and subtropical regions of the globe. The aim of this study was to determine some demographic factors associated with malaria prevalence in Zaria, Kaduna State. A cross sectional hospital based study was conducted on consenting participants in Zaria. Four hundred blood samples were collected, from which Giemsa-stained thick and thin blood films were prepared and examined for the presence of Plasmodium species by microscopy. Demographic data were obtained from all participants using structured questionnaire. Educational status and type of housing were not significantly associated with malaria prevalence; however, the highest prevalence were recorded among those who had only primary education (37.3\%) and those living in Compound houses (29.1). Malaria prevalence was higher among Civil servants (53.8\%) than those in other occupations $(p<0.05)$. Based on marital status, Singles had a prevalence of $35.4 \%$ which was the highest prevalence recorded $(p<0.05)$. Some demographic factors can predispose individuals to mosquito bites, and consequently increase the prevalence of malaria in a population. The occupation and marital status of respondents in this study were significantly associated with malaria prevalence.

Keywords: Malaria, Demographic, Plasmodium, Prevalence, Microscopy

INTRODUCTION

Malaria is a parasitic disease, endemic to Nigeria and many tropical and subtropical regions of the globe. It is caused by the protozoan parasite Plasmodium. Human malaria is caused by four different species of Plasmodium: $P$. falciparum, $P$. malaria, $P$. ovale, $P$. vivax. Occasionally, humans become infected by $P$. knowlesi which normally infects animals (WHO, 2017). It is a perennial public health problem in Sub-Saharan Africa with an enormous global incidence, estimated at 300 million clinical cases each year of which more than one million die (WHO, 1999).

Parasitic diseases pose major obstacles to health growth and socio-economic development in developing countries (Brooker et al., 2007). Parasitic diseases such as malaria are life threatening as well as the leading cause of mortality in endemic countries; more severe to some risky groups, like pregnant women (WACIPAC, 2008).

Demographic factors have been reported to contribute significantly to malaria prevalence (Jepkogei et al., 2016). A clear understanding of the social and behavioral risk factors, and knowledge gaps related to exposure to malaria, are essential when developing guidelines and recommendations for more effective disease prevention in malaria endemic areas (Bashar et al., 2012).This study therefore attempts to provide more information on possible association of some socio-demographic factors with malaria.

\section{MATERIALS AND METHODS}

Study area

The study was carried out in four selected hospitals in Zaria Nigeria; Major Ibrahim B. Abdullahi Memorial Hospital Zaria, Hajiya Gambo Sawaba Hospital Kofan Gaya Zaria, Salama Hospital and St. Luke's Hospital Wusasa Zaria. Zaria is a city in Kaduna state, Nigeria. It is located at 11.11 latitude and 7.72 longitude and it is situated at elevation 644 meters above sea level. Zaria has a population of 975,153 making it the second largest city in Kaduna (World Atlas, 2017).

Sample size

The sample size was determined using a prevalence of $23.45 \%$ (Bechemagbor, 2010) and the following formula as described by Naing et al. (2006): 


$$
\mathrm{n}=\frac{z^{2} p(1-p)}{d^{2}}
$$

$\mathrm{n}=$ number of samples

$\mathrm{p}=$ prevalence rate of previous study $=23.45 \%=0.2345$ (Bechemagbor, 2010)

$z=$ standard normal distribution at $95 \%$ confidence limit $=1.96$

$\mathrm{d}=$ absolute desired precision of $5 \%=0.05$

$z=1.96$

$\mathrm{n}=\frac{1.96^{2 *} 0.2345(1-0.2345)}{0.05^{2}}$

$\mathrm{n}=275$ samples

Four (400) blood samples were however collected for this study.

\section{Administration of structured questionnaire}

The use of a structured questionnaire was employed for the collection of patient's data. Only consenting individuals were enrolled.

\section{Sample collection}

A hundred blood samples were collected from each of the four hospitals, making a total of 400 blood samples altogether. A soft tubing tourniquet was fastened to the upper arm of the patients to enable the index finger to feel a suitable vein. The puncture site was then cleansed with Methylated spirit (methanol) and venipuncture was made with the aid of a $21 \mathrm{G}$ needle attached to a syringe. When sufficient blood was collected, the tourniquet was then released and the needle removed immediately while the blood was transferred into an EDTA bottle (Epidi et al., 2008). The blood was used to make thick and thin blood films according to the method described by Cheesbrough (2009).

Microscopic examination of the stained blood films

The stained blood films were examined under the microscope using 100X objective lens after focusing. Presence of ring forms of Plasmodium and Trophozoites of Plasmodium indicated positive results. A blood smear was considered negative after examining at least 100 high power (100X objective) microscopic fields for parasites (Cheesbrough, 2009). The prevalence rate of malaria was determined as follows:

\section{Ethical Clearance}

$$
\text { Prevalence rate }=\frac{\text { No. } \text { positive }}{\text { Total }} \times 100
$$

Ethical approval and consent was sought and obtained from the ethical committee of Kaduna State Ministry of Health and the ethical committees of the various private hospitals included in the study.
Data analysis

Data generated from the research were analysed using Statistical Package for Social Sciences 20.0 (SPSS Inc., Chicago, USA). Chi square analysis was used to check the level of significance in the occurrence of malaria parasite in relation to different variables at $95 \%$ confidence level.

Results

Table 1 shows malaria prevalence in relation to educational status of respondents. The highest prevalence was recorded among those who had only primary education (37.3\%). Those who went to tertiary institution and those who had other forms of education (Others) had the same prevalence $(33.3 \%)$. The least prevalence $(22.5 \%)$ was seen in those who had no form of education (Nil).

The table 2 shows malaria prevalence in relation to occupation. Civil servants had the highest prevalence $(53.8 \%)$ followed by traders (36.6\%) and artisans (33.3\%). Farmers and respondents who were unemployed had $12.5 \%$ and $27.4 \%$ prevalence respectively. The lowest prevalence was found among those who had other forms of occupations with $10.0 \%$.

Table 3 shows the distribution of malaria according to type of housing. Those who lived in Compound houses had $29.1 \%$ prevalence while those who lived in Self-contain houses had a prevalence of $27.2 \%$. There was no significant difference $(p>0.05)$ in the statistics. The prevalence of malaria according to marital status is also shown in the table 4. Those who were single had the highest prevalence $(35.4 \%)$, followed by married people $(24.3 \%)$ and lastly divorcees $(16 \%)$. The $p$ value $(0.050)$ was statistically significant $(\leq 0.05)$.

Table 1 Malaria prevalence in relation to educational status of respondents

\begin{tabular}{lllll}
\hline Educational status & No. screened & No. positive (\%) & $\mathrm{x}^{2}$ & $\mathrm{P}$ value \\
\hline Tertiary & 135 & $45(33.3)$ & 5.058 & 0.281 \\
Secondary & 106 & $25(23.6)$ & & \\
Primary & 67 & $25(37.3)$ & & \\
Others & 3 & $1(33.3)$ & & \\
Nil & 89 & $20(22.5)$ & & \\
\hline
\end{tabular}


Special Conference Edition, November, 2017

Table 2 Malaria prevalence in relation to occupation

\begin{tabular}{lllll}
\hline Occupation & No. screened & No. positive $(\%)$ & $\mathrm{x}^{2}$ & P value \\
\hline Civil servant & 26 & $14(53.8)$ & 24.566 & 0.000 \\
Trader & 101 & $37(36.6)$ & & \\
Artisan & 9 & $3(33.3)$ & & \\
Farmer & 8 & $1(12.5)$ & & \\
Unemployed & 186 & $51(27.4)$ & & \\
Others & 70 & $7(10.0)$ & & \\
\hline
\end{tabular}

Table 3Malaria prevalence in relation to type of housing

\begin{tabular}{lllll}
\hline Type of housing & No. screened & No. positive (\%) & $\mathrm{x}^{2}$ & P value \\
\hline Self-contain & 180 & $49(27.2)$ & 0.171 & 0.680 \\
Compound house & 220 & $64(29.1)$ & & \\
\hline
\end{tabular}

Table 4 Distribution of malaria according to marital status

\begin{tabular}{lllll}
\hline Marital status & No. screened & No. positive (\%) & $\mathrm{x}^{2}$ & $\mathrm{P}$ value \\
\hline Married & 247 & $60(24.3)$ & 5.987 & 0.050 \\
Single & 147 & $52(35.4)$ & & \\
Divorced & 6 & $1(16.7)$ & & \\
\hline
\end{tabular}

\section{DISCUSSION}

In this study, those with only primary education had a slightly higher prevalence than those with tertiary education. Those without any education at all had the lowest prevalence. This is probably so because majority of the respondents who had no education at all were infants and children who were yet to begin their primary education. It may also be because these infants are more likely to sleep under insecticidal nets than adults (Syed et al., 2011). According to the report of Syed et al. (2011), there has been a substantial improvement in possession and usage of insecticidal bed nets especially for the two most vulnerable groups (under-five children and pregnant women).

Civil servants had the highest prevalence in this study. This could be because there were many respondents in this category whose nature of jobs exposed them to mosquito bites. It could also be as a result of the fact that majority of the civil servants in this study were males, and many studies have reported a higher prevalence of malaria in males than females. This is in agreement with Idoko et al. (2015).

From this study; it was observed that people living in compound houses were more at risk of contracting malaria than those living in selfcontain apartments. This may be due to high hygiene state of most self-contain houses as compared to compound houses. This is in

\section{REFERENCES}

Bashar, K., Al-Amin, H.M., Reza, M. S., Islam, M., Asaduzzaman, Ahmed, T.U. (2012). Socio-demographic factors influencing consonance with the findings of Dawit et al. (2012) who reported a decreased prevalence in houses with better hygiene and facilities than in those without. They also reported that the risk of malaria is higher for households in a lower socio-economic bracket than for those that enjoy a higher status and who are able to afford to take measures to reduce the risk of transmission.

The marital status of respondents in this study was significantly linked to malaria prevalence. The highest prevalence was recorded among those who were single, followed by married people and divorced people.

Conclusion

Some demographic factors can predispose individuals to mosquito bites, and consequently increase the prevalence of malaria in a population. The occupation and marital status of respondents in this study were significantly associated with malaria prevalence. Therefore, there should be increased malaria prevention enlightenment among these groups.

Author's contributions: BGY was responsible for conceptualization, data collection, laboratory analysis, data analysis and interpretation, drafting and revision of the manuscript. KET, MBE and AGS were involved in critical analysis and revision of the manuscript.

Conflict of interest: The authors declare no conflict of interest

knowledge, attitude and practice (KAP) regarding malaria in Bangladesh, $B C M$ Public Health, 12, 1084. 
Bechemagbor, A. (2010). Studies on malaria prevalence, drug treatment regimes and intensity of chloroquine-resistant Pasmodium falciparum in Zaria, Nigeria. Retrieved from http: / /hdl.handle.net/123456789/4048

Brooker, S.J., Akhwale, W., Pullan, R., Estambale, B., Clarke, S.E., Snow R.W., and Hotez, P. (2007). Epidemiology of Plasmodium-Helminth co-infections in Africa: Populations at risk, potential impact on anaemia and prospects for combining control. American Journal of Tropical Medicine and Hygiene, 77(6), 88-98.

Cheesbrough, M. (2009). District laboratory practice in tropical countries part $1\left(2^{\text {nd }}\right.$ edition) New York: Cambridge University Press.

Dawit, G. A., Temesge, T.Z. and Henry, G. M. (2012). Prevalence and risk factors of malaria in Ethiopia. Malaria Journal, $11,195$.

Epidi, T. T., Nwani, C. D., and Ugorji, N. P. (2008). Prevalence of malaria in blood donors in Abakaliki Metropolis, Nigeria, Scientific Research and Essay, 3, 162164.

Idoko, M.O., Ado, S. and Umoh, V. (2015). Prevalence of Dangue virus and Malaria in patients with febrile complaints in Kaduna metropolis, Nigeria, British Research Journal, 8 (1), 343-347.
Jepkogei, M. R., Moses, N. and Makwali, J. (2016). Socio-Demographic Factors Associated with Malaria-Geohelminth Co-Infection and Syndemics in Pregnancy: A Cross Sectional Study of Pregnant Women Attending Ante Natal Care at Nandi Hills Sub County Hospital, Kenya. Journal of Biology, Agriculture and Healthcare, 6 (14), ISSN 2224-3208.

Naing, L., Winn, T. and Rusli, B.N. (2006). Practical issues in calculating the sample size for prevalence studies. Archives of Orofacial Sciences, 1, 9-14.

Syed, M A., Shamim, H., Mohammad, M.K. and Sanjit, R. (2011). Free distribution of insecticidal bed nets improves possession and preferential use by households and is equitable: findings from two cross-sectional surveys in thirteen malaria endemic districts of Bangladesh. Malaria Journal, 10, 357.

WACIPAC (2008). Why parasitic disease control? Available http: / /98.130.228.222/wacipac/index. php, accessed on January 10/2010.

WHO (1999). The World Health Report: Making a difference. (Geneva: World Health Organization.)

WHO (2017). Malaria. Retrieved $5^{\text {th }}$ July 2017 , from:

http://www. who.int/ith/diseases/mala ria/en/

World Atlas (2017). Retrieved $5^{\text {th }}$ July 2017, from www.worldatlas.com 